\title{
The Abel-type polynomial identities*
}

\author{
Fengying Huang
}

School of Mathematical Sciences, South China Normal University ; School of Computer Science, Guangdong Polytechnic Normal University,

Guangzhou, 510631, P.R. China.

E-mail:hfy.ing@163.com

Bolian Liu

School of Mathematical Sciences, South China Normal University, Guangzhou, 510631, P.R. China.

Corresponding author.

E-mail:liubl@scnu.edu.cn

Submitted: Sep 23, 2009; Accepted: Dec 29, 2009; Published: Jan 5, 2010

Mathematics Subject Classification: 05C30; 05C05

\begin{abstract}
The Abel identity is $(x+y)^{n}=\sum_{i=0}^{n}\left(\begin{array}{c}n \\ i\end{array}\right) x(x-i z)^{i-1}(y+i z)^{n-i}$, where $x, y$ and $z$ are real numbers. In this paper we deduce several polynomials expansions, referred to as Abel-type identities, by using Foata's method, and also show some of their applications.
\end{abstract}

\section{Introduction}

It is well-known that the binomial identity is $(x+y)^{n}=\sum_{i=0}^{n}\left(\begin{array}{c}n \\ i\end{array}\right) x^{i} y^{n-i}$. In 1826, Abel deduced an identity which is

$$
(x+y)^{n}=\sum_{i=0}^{n}\left(\begin{array}{l}
n \\
i
\end{array}\right) x(x-i z)^{i-1}(y+i z)^{n-i},
$$

where $x, y$ and $z$ are real numbers. Then the identity is called Abel identity. When we set $z=0$ in Eq.(1), it becomes the binomial identity. There are many applications of the Abel identity [1]. And many authors offered different proofs of this identity, including the

${ }^{*}$ Supported by NNSF of China(No.10771080). 
elegant combinatorial methods by Foata [2], the algebraic method by Lucas [1] and the coding sign method by Francon [1]. In 1996, S.B.Ekhad and J.E.Majewicz presented a computer-generated proof of it [3].

Another well-known version of the classical Abel identity [4] is

$$
(x+y+n z)(x+y)^{n-1}=\sum_{i=0}^{n}\left(\begin{array}{l}
n \\
i
\end{array}\right) x \cdot(x-i z)^{i-1}(y+n z)(y+i z)^{n-i-1},
$$

while a generalization of Abel identity expanding a product of multivariate linear forms is Hurwitz identity [1] which is

$$
\begin{aligned}
& (x+y)\left(x+y+z_{1}+z_{2}+\cdots+z_{n}\right)^{n-1}=\sum\left\{x \left(x+\varepsilon_{1} z_{1}+\varepsilon_{2} z_{2}+\cdots\right.\right. \\
& \left.\left.+\varepsilon_{n} z_{n}\right)^{\varepsilon_{1}+\varepsilon_{2}+\cdots+\varepsilon_{n}-1} y\left(y+\bar{\varepsilon}_{1} z_{1}+\bar{\varepsilon}_{2} z_{2}+\cdots+\bar{\varepsilon}_{n} z_{n}\right)^{\bar{\varepsilon}_{1}+\bar{\varepsilon}_{2}+\cdots+\bar{\varepsilon}_{n}-1}\right\}
\end{aligned}
$$

where the sum is over all $2^{n}$ possibilities with $\varepsilon_{1}, \varepsilon_{2}, \cdots, \varepsilon_{n}$ choosing 0 or 1 and $\bar{\varepsilon}_{i}=1-\varepsilon_{i}$, $(i=1,2, \cdots, n)$.

All the identities above are dealt with a single summation. In this paper we present three polynomial identities, which are called the Abel-type identities involving double

summations. We show the identities here first and then give their proofs in the third Section.

Theorem 1.1 (The Abel-type identities) Assume that $0^{0}=1$. For any real numbers $x$, $y, z$ and $u$, the following identities hold.

(1) $(x+y)^{m} u^{n}=\sum_{i=0}^{n} \sum_{j=0}^{m}\left(\begin{array}{c}n \\ i\end{array}\right)\left(\begin{array}{c}m \\ j\end{array}\right) x(x-i z)^{j-1}(y+i z)^{m-j}(-j z)^{i}(u+j z)^{n-i}$.

$$
\begin{aligned}
& (x+y+n z)(-m z)^{n}(x+y)^{m-1} \\
= & \sum_{i=0}^{n} \sum_{j=0}^{m}\left(\begin{array}{c}
n \\
i
\end{array}\right)\left(\begin{array}{c}
m \\
j
\end{array}\right) x(x-i z)^{j-1}(y+n z)(y+i z)^{m-j-1}(-j z)^{i}(-m z+j z)^{n-i} . \\
& {\left[(x+y) u-n m z^{2}\right](x+y)^{m-1} u^{n-1}=\sum_{i=0}^{n} \sum_{j=0}^{m}\left\{\left(\begin{array}{c}
n \\
i
\end{array}\right)\left(\begin{array}{c}
m \\
j
\end{array}\right)(x+y+n z)\right.} \\
\times & \left.(x+y+n z-i z)^{j-1}(-n z+i z)^{m-j}(u+m z)(-j z)^{i}(u+j z)^{n-i-1}\right\} .
\end{aligned}
$$

In this paper, firstly, we introduce the coding method, due to Foata ( $\$ 1.18$ of [1]). Next by using this method, we give the proof of Theorem 1.1. At last some applications of Theorem 1.1 are presented, i.e., the identities (2) and (3) helping for enumerating the spanning forests of complete bipartite graph.

\section{Preliminaries}

In this section, we will introduce some terminologies which can be found in [1].

Suppose $[n]$ denote a set with $n$ elements, i.e., $[n]=\{1,2, \cdots, n\} .[n]^{[n]}$ is a set containing all mappings from $[n]$ to $[n]$. 
Given a subset $E$ of $[n]^{[n]}$, we define the (commutative) coding polynomial of $E$ as $\mathcal{T}_{E}=\mathcal{T}_{E}\left(t_{1}, t_{2}, \ldots\right)=\sum_{f \in E} t(f)$. If $f$ maps $\alpha_{i}$ elements to $i(i=1,2, \cdots, n), t(f)=$ $t_{1}^{\alpha_{1}} t_{2}^{\alpha_{2}} \cdots t_{n}^{\alpha_{n}}$. Then the coefficient of $t_{1}^{\alpha_{1}} t_{2}^{\alpha_{2}} \cdots$ in $\mathcal{T}_{E}\left(t_{1}, t_{2}, \cdots\right)$ is the number of $f \in E$ such that $f$ maps $\alpha_{i}$ elements to $i(i=1,2, \cdots, n)$. Evidently, $\mathcal{T}_{E}(1,1, \cdots)=|E|$, i.e., the number of elements of the set $E$.

Take $E \subseteq[3]^{[3]}$ for example, where $E=\left\{f_{1}, f_{2}, f_{3}, f_{4}\right\}$ with $f_{1}(i)=1$, for $i=1,2,3$; $f_{2}(1)=2, f_{2}(2)=2, f_{2}(3)=1 ; f_{3}(1)=2, f_{3}(2)=3, f_{3}(3)=1 ;$ and $f_{4}(1)=2$, $f_{4}(2)=1, f_{4}(3)=2$. We have $t\left(f_{1}\right)=t_{1}^{3}, t\left(f_{2}\right)=t\left(f_{4}\right)=t_{1} t_{2}^{2}$ and $t\left(f_{3}\right)=t_{1} t_{2} t_{3}$. And thus $\mathcal{T}_{E}=t_{1}^{3}+2 t_{1} t_{2}^{2}+t_{1} t_{2} t_{3}$, and $\mathcal{T}_{E}(1,1,1)=4=|E|$.

We present the following results about $\mathcal{T}_{E}$ here for they can help to prove Theorem 1.1 .

Result 1 Set $E=[n]^{[n]}$. Then $\mathcal{T}_{E}=\left(t_{1}+t_{2}+\cdots+t_{n}\right)^{n}$.

Result 2 If $E$ is the set containing all functions fixed at $1,2, \cdots, k, \mathcal{T}_{E}=t_{1} t_{2} \cdots t_{k}\left(t_{1}+\right.$ $\left.t_{2}+\cdots+t_{n}\right)^{n-k}$.

Result 3 If $E$ is the set which contains all acyclic functions rooted or fixed at $1,2, \cdots, k$, then $\mathcal{T}_{E}=t_{1} t_{2} \ldots t_{k}\left(t_{1}+t_{2}+\cdots+t_{k}\right)\left(t_{1}+t_{2}+\cdots+t_{n}\right)^{n-k-1}$.

Thus, we have (1) $\left|[n]^{[n]}\right|=n^{n}$; (2) the number of functions fixed at $k$ given elements is $n^{n-k} ;(3)$ the number of forests rooted at $k$ given vertices is $k \cdot n^{n-k-1}$.

Some properties of $\mathcal{T}_{E}$ can be deduced.

Property 2.1 If $E$ can be separated into different types $E_{1}, E_{2}, \cdots$, written as $E=$ $E_{1}+E_{2}+\cdots$, then $\mathcal{T}_{E}=\mathcal{T}_{E_{1}}+\mathcal{T}_{E_{2}}+\cdots$.

Property 2.2 For any $f \in E$, if there exist $f_{i} \in E_{i}(i=1,2, \cdots)$ such that $f=f_{1} f_{2} \cdots$, i.e. $E=E_{1} E_{2} \cdots$, then $\mathcal{T}_{E}=\mathcal{T}_{E_{1}} \mathcal{T}_{E_{2}} \cdots$.

\section{Proof of Theorem 1.1}

We consider the enumeration of function sets as follows:

(I) The number of elements of the function set $E_{1} \subseteq[n+m+4]^{[n+m+4]}$ which contains all functions fixed at $n+1, n+2, n+m+3$ and $n+m+4$ such that $f$ maps $[n]$ to $[n+m+4]-[n+2]$ while $[n+m+2]-[n+2]$ to $[n+2]$ for any $f \in E_{1}$.

(II) The number of elements of the function set $E_{2} \subseteq[n+m+2]^{[n+m+2]}$ which contains all acyclic functions fixed or rooted at $n+1$ and $n+2$ such that $f$ maps $[n]$ to $[n+m+$ $2]-[n+2]$ while $[n+m+2]-[n+2]$ to $[n+2]$ for any $f \in E_{2}$.

(III) The number of elements of the function set $E_{3} \subseteq[n+m+4]^{[n+m+4]}$ which contains all acyclic functions fixed or rooted at $n+1, n+2, n+m+3$ and $n+m+4$ such that $f$ maps $[n]$ to $[n+m+4]-[n+2]$ while $[n+m+2]-[n+2]$ to $[n+2]$ for any $f \in E_{3}$.

Now we will obtain the coding polynomials $\mathcal{T}_{E_{i}}$, where $E_{i}(i=1,2,3)$ are defined as above.

(I) From Result 2 and Property 2.2, the following result holds

$$
\mathcal{T}_{E_{1}}=t_{n+1} t_{n+2}\left(t_{n+3}+t_{n+4}+\cdots+t_{n+m+4}\right)^{n} t_{n+m+3} t_{n+m+4}\left(t_{1}+t_{2}+\cdots+t_{n+2}\right)^{m} .
$$


Let $X \subseteq[n], Y \subseteq[n+m+2]-[n+2],|X|=i$ and $|Y|=j$. Set $\bar{X}=[n]-X$, $\bar{Y}=([n+m+2]-[n+2])-Y$. Consequently, $0 \leqslant i \leqslant n, 0 \leqslant j \leqslant m,|\bar{X}|=n-i$ and $|\bar{Y}|=m-j$. Set $A_{1}=X \cup\{n+1, n+2\} \cup Y$ and $A_{2}=\bar{X} \cup\{n+m+3, n+m+4\} \cup \bar{Y}$. Let $E_{1}^{(1)}(X, Y) \subseteq A_{1}^{A_{1}}$ be a set containing all acyclic functions rooted at $n+1$ and $n+2$, and $E_{1}^{(2)}(X, Y) \subseteq A_{2}^{A_{2}}$ be a set containing all functions rooted at $n+m+3$ and $n+m+4$. Thus $E_{1}(X, Y)=E_{1}^{(1)}(X, Y) E_{1}^{(2)}(X, Y)$, and combining Result 2, Result 3 and Property 2.2 , we have

$$
\begin{aligned}
\mathcal{T}_{E_{1}(X, Y)}= & \mathcal{T}_{E_{1}^{(1)}(X, Y)} \mathcal{T}_{E_{1}^{(2)}(X, Y)} \\
= & t_{n+1} t_{n+2}\left(\sum_{q \in Y} t_{q}\right)^{|X|}\left(t_{n+1}+t_{n+2}\right)\left(t_{n+1}+t_{n+2}+\sum_{p \in X} t_{p}\right)^{|Y|-1} \\
& \times\left(t_{n+m+3}+t_{n+m+4}+\sum_{q \in \bar{Y}} t_{q}\right)^{|\bar{X}|} t_{n+m+3} t_{n+m+4}\left(\sum_{p \in \bar{X}} t_{p}\right)^{|\bar{Y}|}
\end{aligned}
$$

From Eq.(2) and Eq.(3), we have

$$
\begin{aligned}
= & \left(t_{n+3}+t_{n+4}+\cdots+t_{n+m+4}\right)^{n}\left(t_{1}+t_{2}+\cdots+t_{n+2}\right)^{m} . \\
& \sum_{X, Y}\left\{\left(\sum_{q \in Y} t_{q}\right)^{|X|}\left(t_{n+1}+t_{n+2}\right)\left(t_{n+1}+t_{n+2}+\sum_{p \in X} t_{p}\right)^{|Y|-1}\right. \\
& \left.\times\left(t_{n+m+3}+t_{n+m+4}+\sum_{q \in \bar{Y}} t_{q}\right)^{n-|X|}\left(\sum_{p \in \bar{X}} t_{p}\right)^{m-|Y|}\right\},
\end{aligned}
$$

where the sum is over all subsets $X \subseteq[n]$ and $Y \subseteq[n+m+2]-[n+2]$.

(II) We have

$$
\mathcal{T}_{E_{2}}=t_{n+1} t_{n+2}\left(t_{n+3}+t_{n+4}+\cdots+t_{n+m+2}\right)^{n}\left(t_{1}+t_{2}\right)\left(t_{1}+t_{2}+\cdots+t_{n+2}\right)^{m-1} .
$$

Choose $X, Y, \bar{X}$ and $\bar{Y}$ the same as in case (I). Set $A_{1}=X \cup\{n+1\} \cup Y$ and $A_{2}=$ $\bar{X} \cup\{n+2\} \cup \bar{Y}$. Let $E_{2}^{(1)}(X, Y) \subseteq A_{1}^{A_{1}}$ be a set containing all acyclic functions rooted at $n+1$, and $E_{2}^{(2)}(X, Y) \subseteq A_{2}^{A_{2}}$ be a set containing all acyclic functions rooted at $n+2$. Thus $E_{2}(X, Y)=E_{2}^{(1)}(X, Y) E_{2}^{(2)}(X, Y)$ and

$$
\begin{aligned}
& \mathcal{T}_{E_{2}(X, Y)}=\mathcal{T}_{E_{2}^{(1)}(X, Y)} \mathcal{T}_{E_{2}^{(2)}(X, Y)} \\
= & t_{n+1}\left(\sum_{q \in Y} t_{q}\right)^{|X|} t_{n+1}\left(t_{n+1}+\sum_{p \in X} t_{p}\right)^{|Y|-1} t_{n+2}\left(\sum_{q \in \bar{Y}} t_{q}\right)^{|\bar{X}|} t_{n+2}\left(t_{n+2}+\sum_{p \in \bar{X}} t_{p}\right)^{|\bar{Y}|-1} .
\end{aligned}
$$

Combining Eq.(5) and Eq.(6), we obtain the following identity.

$$
=\begin{aligned}
& \left(t_{1}+t_{2}\right)\left(t_{n+3}+t_{n+4}+\cdots+t_{n+m+2}\right)^{n}\left(t_{1}+t_{2}+\cdots+t_{n+2}\right)^{m-1} \\
& \sum_{X, Y}\left(\sum_{q \in Y} t_{q}\right)^{|X|} t_{n+1}\left(t_{n+1}+\sum_{p \in X} t_{p}\right)^{|Y|-1}\left(\sum_{q \in \bar{Y}} t_{q}\right)^{n-|X|} t_{n+2}\left(t_{n+2}+\sum_{p \in \bar{X}} t_{p}\right)^{m-|Y|-1},
\end{aligned}
$$

where the sum is over all subsets $X \subseteq[n]$ and $Y \subseteq[n+m+2]-[n+2]$.

(III) Define a point $v \in[n+m+4]$ to be isolated provided that there exists no elements mapping onto it except itself. By the definition of $E_{3}$, the possible isolated points may be and only may be the root points $n+1, n+2, n+m+3$ and $n+m+4$. Suppose 
$E_{3}^{(1)}, E_{3}^{(2)}, E_{3}^{(3)} \subseteq E_{3}$, where $E_{3}^{(1)}$ contains all acyclic functions whose possible isolatedpoint sets are $\{n+1\},\{n+2\},\{n+1, n+m+3\},\{n+2, n+m+3\},\{n+1, n+m+4\}$, $\{n+2, n+m+4\},\{n+m+3, n+m+4\},\{n+1, n+m+3, n+m+4\},\{n+2, n+m+3, n+m+4\}$ and $\emptyset ; E_{3}^{(2)}$ contains all acyclic functions whose possible isolated-point sets are $\{n+m+3\}$, $\{n+m+4\},\{n+1, n+m+3\},\{n+2, n+m+3\},\{n+1, n+m+4\},\{n+2, n+m+4\}$, $\{n+1, n+2\},\{n+1, n+2, n+m+3\},\{n+1, n+2, n+m+4\}$ and $\emptyset$; while $E_{3}^{(3)}$ contains all acyclic functions whose possible isolated-point sets are $\{n+1, n+m+3\}$, $\{n+1, n+m+4\},\{n+2, n+m+3\},\{n+2, n+m+4\}$ and $\emptyset$. Note that both $n$ and $m$ are positive. It is impossible that $\{n+1, n+2, n+m+3, n+m+4\}$ is an isolated-point set of $E_{3}$. Therefore $E_{3}=E_{3}^{(1)}+E_{3}^{(2)}-E_{3}^{(3)}$. And thus $\mathcal{T}_{E_{3}}=\mathcal{T}_{E_{3}^{(1)}}+\mathcal{T}_{E_{3}^{(2)}}-\mathcal{T}_{E_{3}^{(3)}}$. However, by Result 2, Result 3 and Property 2.2, we have

$$
\begin{aligned}
\mathcal{T}_{E_{3}^{(1)}}= & t_{n+m+3} t_{n+m+4}\left(t_{n+1}+t_{n+2}\right)\left(t_{1}+t_{2}+\cdots+t_{n+2}\right)^{m-1} t_{n+1} t_{n+2} \\
& \times\left(t_{n+3}+t_{n+4}+\cdots+t_{n+m+4}\right)^{n} \\
\mathcal{T}_{E_{3}^{(2)}=} & t_{n+1} t_{n+2}\left(t_{n+m+3}+t_{n+m+4}\right)\left(t_{n+3}+t_{n+4}+\cdots+t_{n+m+4}\right)^{n-1} \\
& \times t_{n+m+3} t_{n+m+4}\left(t_{1}+t_{2}+\cdots+t_{n+2}\right)^{m}
\end{aligned}
$$

and

$$
\begin{aligned}
\mathcal{T}_{E_{3}^{(3)}}= & t_{n+1} t_{n+2}\left(t_{n+m+3}+t_{n+m+4}\right)\left(t_{1}+t_{2}+\cdots+t_{n+2}\right)^{m-1} t_{n+m+3} \\
& \times t_{n+m+4}\left(t_{n+1}+t_{n+2}\right)\left(t_{n+3}+t_{n+4}+\cdots+t_{n+m+4}\right)^{n-1} .
\end{aligned}
$$

On the other hand, we choose $X, Y, \bar{X}$ and $\bar{Y}$ the same as in the case (I). Set $A_{1}=X \cup\{n+1, n+2\} \cup Y$ and $A_{2}=\bar{X} \cup\{n+m+3, n+m+4\} \cup \bar{Y}$. Let $E_{3}^{(1)}(X, Y) \subseteq A_{1}^{A_{1}}$ be a set containing all acyclic functions rooted at $n+1$ and $n+2$, and $E_{3}^{(2)}(X, Y) \subseteq A_{2}^{A_{2}}$ be a set containing all acyclic functions rooted at $n+m+3$ and $n+m+4$. Thus $E_{3}(X, Y)=E_{3}^{(1)}(X, Y) E_{3}^{(2)}(X, Y)$ and then it yields that

$$
\begin{aligned}
& \mathcal{T}_{E_{3}(X, Y)}=\mathcal{T}_{E_{3}^{(1)}(X, Y)} \mathcal{T}_{E_{3}^{(2)}(X, Y)} \\
= & t_{n+1} t_{n+2}\left(\sum_{q \in Y} t_{q}\right)^{|X|}\left(t_{n+1}+t_{n+2}\right)\left(t_{n+1}+t_{n+2}+\sum_{p \in X} t_{p}\right)^{|Y|-1} t_{n+m+3} \\
& \times t_{n+m+4}\left(\sum_{p \in \bar{X}} t_{p}\right)^{|\bar{Y}|}\left(t_{n+m+3}+t_{n+m+4}\right)\left(t_{n+m+3}+t_{n+m+4}+\sum_{q \in \bar{Y}} t_{q}\right)^{|\bar{X}|-1},
\end{aligned}
$$

where $X \subseteq[n],|X|=i, \bar{X}=[n]-X, Y \subseteq[n+m+2]-[n+2],|Y|=j$ and $\bar{Y}=[n+m+2]-[n+2]-Y$.

Thus we obtain the following equation.

$$
\begin{aligned}
& \left(t_{1}+\cdots+t_{n+2}\right)^{m-1}\left(t_{n+3}+\cdots+t_{n+m+2}\right)^{n-1}\left[( t _ { n + 1 } + t _ { n + 2 } ) \left(t_{n+3}+\ldots\right.\right. \\
& \left.\left.+t_{n+m+4}\right)+\left(t_{n+m+3}+t_{n+m+4}\right)\left(t_{1}+\cdots+t_{n+2}\right)\right] \\
= & \sum_{i=0}^{n} \sum_{j=0}^{m}\left(\begin{array}{c}
n \\
i
\end{array}\right)\left(\begin{array}{c}
m \\
j
\end{array}\right)\left\{\left(t_{n+1}+t_{n+2}\right)\left(t_{n+1}+t_{n+2}+\sum_{p \in X} t_{p}\right)^{j-1}\left(\sum_{p \in \bar{X}} t_{p}\right)^{m-j}\right. \\
& \left.\times\left(t_{n+m+3}+t_{n+m+4}\right)\left(\sum_{q \in Y} t_{q}\right)^{i}\left(t_{n+m+3}+t_{n+m+4}+\sum_{q \in \bar{Y}} t_{q}\right)^{n-i-1}\right\} .
\end{aligned}
$$


Set $t_{n+1}=x, \quad t_{n+2}=y+n z, \quad t_{1}=t_{2}=\cdots=t_{n}=-z, t_{n+m+3}=u, \quad t_{n+m+4}=m z$, and $t_{n+3}=t_{n+4}=\cdots=t_{n+m+2}=-z$ in Eqs.(7), (8) and (4), respectively. We obtain identities (2), (3) of Theorem 1.1 and

$$
\begin{aligned}
& (x+y)^{m} u^{n}=\sum_{i=0}^{n} \sum_{j=0}^{m}\left\{\left(\begin{array}{c}
n \\
i
\end{array}\right)\left(\begin{array}{c}
m \\
j
\end{array}\right)(x+y+n z)\right. \\
& \left.\times(x+y+n z-i z)^{j-1}(-n z+i z)^{m-j}(-j z)^{i}(u+j z)^{n-i}\right\},
\end{aligned}
$$

respectively. And then by replacing $x$ with $x+y+n z$, and $y$ with $-n z$ in Eq.(9), the identity (1) of Theorem 1.1 is obtained. Thus Theorem 1.1 is proved.

Suppose $k$ and $l$ be positive integers. Replace $n$ by $n-k$ and let $z=-1, x=s$ and $y=n-s$ in identities (1) and (2), and then replace $n$ and $m$ by $n-k$ and $m-l$, respectively, and let $z=-1, x+y=n$ and $u=m$ in identities (1) and (3) of Theorem 1.1. We obtain three interesting identities as follows:

\section{Theorem 3.1}

$$
\begin{aligned}
m^{n-k} n^{m-l}= & \sum_{i=0}^{n-k} \sum_{j=0}^{m-l}\left(\begin{array}{c}
n-k \\
i
\end{array}\right)\left(\begin{array}{c}
m-l \\
j
\end{array}\right) s(s+i)^{j-1}(n-s-i)^{m-l-j} j^{i}(m-j)^{n-k-i}, \\
& k \cdot m^{n-k} n^{m-1} \\
= & \sum_{i=0}^{n-k} \sum_{j=0}^{m}\left(\begin{array}{c}
n-k \\
i
\end{array}\right)\left(\begin{array}{c}
m \\
j
\end{array}\right) s(s+i)^{j-1}(k-s)(n-s-i)^{m-j-1} j^{i}(m-j)^{n-k-i}
\end{aligned}
$$

and

$$
\begin{aligned}
& (k m+l n-k l) \cdot m^{n-k-1} n^{m-l-1} \\
= & \sum_{i=0}^{n-k} \sum_{j=0}^{m-l}\left(\begin{array}{c}
n-k \\
i
\end{array}\right)\left(\begin{array}{c}
m-l \\
j
\end{array}\right) k l(k+i)^{j-1}(n-k-i)^{m-j} j^{i}(m-j)^{n-k-i-1},
\end{aligned}
$$

where $0^{0}=1$ and $1 \leqslant s \leqslant k$ in Eq.(10) or $1 \leqslant s \leqslant k-1$ in Eq.(11) is an integer.

\section{Applications}

Let $K_{m, n}$ be a labeled complete bipartite graph with vertex set $V\left(K_{m, n}\right)=A \cup B,|A|=m$, $|B|=n$. A forest of $l+k$ labeled rooted trees as spanning subgraphs of $K_{m, n}$ with $l$ roots in $A$ and $k$ roots in $B$ is denoted by $[m, l ; n, k]$ - forests $(l \leqslant m, k \leqslant n)$ while the number of $[m, l ; n, k]$-forests is denoted by $f(m, l ; n, k)$.

In [5], Y. Jin and C. Liu obtained the following results.

Theorem A For $m \geqslant 0, n \geqslant 1$ and $k \geqslant 1$,

$$
f(m, 0 ; n, k)=k\left(\begin{array}{l}
n \\
k
\end{array}\right) m^{n-k} n^{m-1}=\left(\begin{array}{c}
n-1 \\
k-1
\end{array}\right) m^{n-k} n^{m},
$$

where $f(0,0 ; 1,1)$ is defined to be 1 .

Theorem B For $1 \leqslant l \leqslant m$ and $1 \leqslant k \leqslant n$,

$$
f(m, l ; n, k)=\left(\begin{array}{c}
m \\
l
\end{array}\right)\left(\begin{array}{l}
n \\
k
\end{array}\right) n^{m-l-1} m^{n-k-1}(k m+l n-k l) .
$$


Let $[m, l ; n, k]^{*}$ - forest denote $[m, l ; n, k]$-forest with $l$ fixed roots in $A$ and $k$ fixed roots in $B$. Similarly, $f^{*}(m, l ; n, k)$ denotes the number of $[m, l ; n, k]^{*}$-forests.

It is easy to know that $f(m, l ; n, k)=\left(\begin{array}{c}m \\ l\end{array}\right)\left(\begin{array}{c}n \\ k\end{array}\right) f^{*}(m, l ; n, k)$. Combining Theorem $A$ and $B$, we have $f^{*}(m, 0 ; n, k)=k m^{n-k} n^{m-1}$ and $f^{*}(m, l ; n, k)=n^{m-l-1} m^{n-l-1}(k m+l n-k l)$.

For the applications of Theorem 3.1, Eqs.(11) and (12) can be used to prove the enumerations of $[m, 0 ; n, k]^{*}$-forests and $[m, l ; n, k]^{*}$-forests, respectively .

In fact, we have the following recurrences

$$
\begin{aligned}
f^{*}(m, 0 ; n, k)=\sum_{i=0}^{n-k} \sum_{j=0}^{m}\left(\begin{array}{c}
n-k \\
i
\end{array}\right)\left(\begin{array}{c}
m \\
j
\end{array}\right) f^{*}(j, 0 ; i+1, s) f^{*}(m-j, 0 ; n-i-1, k-s) . \\
f^{*}(m, l ; n, k)=\sum_{i=0}^{n-k} \sum_{j=0}^{m-l}\left(\begin{array}{c}
n-k \\
i
\end{array}\right)\left(\begin{array}{c}
m-l \\
j
\end{array}\right) f^{*}(j, 0 ; k+i, k) f^{*}(m-j, l ; n-k-i, 0) \\
=\sum_{i=0}^{n-k} \sum_{j=0}^{m-l}\left(\begin{array}{c}
n-k \\
i
\end{array}\right)\left(\begin{array}{c}
m-l \\
j
\end{array}\right) f^{*}(j, 0 ; k+i, k) f^{*}(n-k-i, 0 ; m-j, l) .
\end{aligned}
$$

From these two recurrences and applying Theorem 3.1, we can prove Theorem $A$ and Theorem $B$ by induction, respectively and thus give another proofs for them.

Note: If we set $t_{i}=1$ in Case (II) and (III) as above, we see that:

In Case (II), it enumerates $[m, 0 ; n+2,2]^{*}$-forests;

In Case (III), it enumerates $[m+2,2 ; n+2,2]^{*}$-forests.

Even so, we can use the results of Case (II) and (III) to enumerate $[m, 0 ; n, k]^{*}$-forests and $[m, l ; n, k]^{*}$-forests, respectively. That's what the Foata's coding method does.

\section{Acknowledgements}

The authors would like to thank the referees for carefully reading and giving many helpful suggestions.

\section{References}

[1] L. Comtet, Advanced Combinatorics, D.Reidel Publ. Co., Dordrechet/Boston, 1974.

[2] D. Foata, Enumerating $k$-Trees, Discr. Math. 1(1971), 181-186.

[3] S. B. Ekhad and J.E. Majewicz, A short WZ-style proof of Abel's identity, Elect. J. Comb. 3(2)(1996): $\sharp R 16,1$.

[4] J. Riordan, Combinatorial identities. John Wiley and Sons, New York, 1968.

[5] Y.L. Jin and C.L. Liu, Enumeration for spanning forests of complete bipartite graphs, ARS Combinatoria, Vol LXX(2004), 85-88.

[6] C. J. Liu and Y. Chow, Enumeration of forests in a graph, Proc. A.M.S. 83(3)(1981), 659-662. 